\title{
Big islands in dispersing billiard-like potentials
}

\author{
Vered Rom-Kedar ${ }^{1, \mathrm{a}, *}$, Dmitry Turaev ${ }^{\mathrm{b}}$ \\ ${ }^{a}$ The Department of Applied Mathematics and Computer Science, The Weizmann Institute of Science, P.O. Box 26, Rehovot 76100, Israel \\ ${ }^{\mathrm{b}}$ Weierstrass Institute for Applied Analysis and Stochastics, Mohrenstr. 39, 10117 Berlin, Germany \\ Received 6 April 1998; received in revised form 22 January 1999; accepted 28 January 1999 \\ Communicated by C.K.R.T. Jones
}

\begin{abstract}
We derive a rigorous estimate of the size of islands (in both phase space and parameter space) appearing in smooth Hamiltonian approximations of scattering billiards. The derivation includes the construction of a local return map near singular periodic orbits for an arbitrary scattering billiard and for the general smooth billiard potentials. Thus, universality classes for the local behavior are found. Moreover, for all scattering geometries and for many types of natural potentials which limit to the billiard flow as a parameter $\epsilon \rightarrow 0$, islands of polynomial size in $\epsilon$ appear. This suggests that the loss of ergodicity via the introduction of the physically relevant effect of smoothening of the potential in modeling, for example, scattering molecules, may be of physically noticeable effect. ()1999 Elsevier Science B.V. All rights reserved.
\end{abstract}

MSC: $58 \mathrm{~F} 15 ; 82 \mathrm{C} 05 ; 34 \mathrm{C} 37 ; 58 \mathrm{~F} 05 ; 58 \mathrm{~F} 13 ; 58 \mathrm{~F} 14$

Keywords: Singular Hamiltonian systems; Elliptic islands; Ergodicity; Mixed dynamics

\section{Introduction}

When an integrable Hamiltonian system is perturbed, non-integrable motion appears [1]. What happens when a highly chaotic Hamiltonian system is perturbed? In particular, consider an ergodic mixing flow - can a perturbation ruin these properties? Clearly, for uniformly hyperbolic systems the answer is negative, e.g., for geodesic flows on the surfaces of negative curvature [2]. The other basic example for hyperbolic behavior in the Hamiltonian setting is the class of scattering billiards [3]; Billiard motion corresponds to a point particle traveling with a constant speed in a region, undergoing elastic collisions at the region's boundary. When the boundary is concave, causing neighboring trajectories to diverge upon reflection, the billiard is called scattering. Due to the divergence instability the scattering billiards are ergodic and mixing systems [3-5]. Thus, these have been suggested [3] as a first step

\footnotetext{
*Corresponding author. Tel.: +1-212-998-3251; fax: +1-212-995-4121; e-mail: vered@ cims.nyu.edu.

${ }^{1}$ Present address; Courant Institution of Mathematical Science, 251 Mercer Street, New York University, New York, NY 10012-1185, USA.
} 
models for substantiating the basic assumption of statistical mechanics - the ergodic hypothesis of Boltzmann (see especially the discussion and references in $[4,6])$.

The scattering billiard corresponds to a non-smooth dynamical system which may formally be thought of as a Hamiltonian motion in a singular potential. However, classical molecules move in smooth (though very steep) potentials. Therefore, it is natural to consider the effect of smoothening of the potential on scattering billiards, and in particular its effect on the ergodic properties. In [7] we proved that smooth scattering billiard-like potentials may give rise to islands, ruining ergodicity (see also [8] for related results concerning the 'finite range potentials' problem). Thus, we demonstrated that proving Boltzmann hypothesis for the hard sphere model is a priori insufficient for proving the achievement of thermodynamical equilibrium for a gas.

Islands appearing in highly chaotic regimes were observed in numerous numerical simulations $[9,10]$. While many of these simulations were carried for the standard map, only recently it has been shown that for large $K$ it has a dense set of parameter values for which elliptic islands exist [11]. It is tempting to assume that the numerically observed chaotic sea is a nonuniformly hyperbolic set of positive measure; however, no results in this direction have appeared to the best of our knowledge.

What is clearly known and has, thus, attracted much attention [9,12-16] is that the appearance of islands in large 'chaotic sea' is of vast significance; First, it implies that the tail of the correlation function has finite range oscillations for initial conditions falling in the island. Moreover, around the islands there may be a 'stickiness' region, which influences the temporal correlation function of initial conditions from the 'chaotic sea'. It is suspected that this stickiness may cause power-law instead of exponential decay of correlations even when the initial conditions lie in the chaotic sea.

Islands may be useful; When living in a chaotic sea, the islands correspond to stable dynamics in a highly noisy environment. This observation may be used in the context of control of chaos (see focus issue in [16]), where it is desirable to switch quickly from highly ordered to highly chaotic behavior. Our construction enables to locate islands in both phase space and parameter space of the smooth billiard-like flow, allowing for a control of such switching.

In all applications the size of the islands and their periodicity matters; these define the space and time scales on which the transition from chaotic to periodic motion is observable. For example, consider the time correlation function

$$
C(T ; x(0))=\lim _{\tau \rightarrow \infty} \frac{1}{2 \tau} \int_{-\tau}^{\tau} f(x(t)) f(x(t+T)) \mathrm{d} t .
$$

This function will essentially be $N$-periodic for $x(0) \in I_{N}$ where $I_{N}$ denotes an island of period $N$. Thus, clearly, the difference between the chaotic sea and island behavior will not be seen before time of order $N$ - demonstrating the crucial dependence on the periodicity of the islands. Averaging Eq. (1) over $x(0)$ gives the correlation function for a general ensemble of initial conditions - clearly the contribution of the time correlation function associated with the island is proportional to its area.

Here, we consider a two degrees of freedom Hamiltonian flow associated with

$$
H=\frac{p_{x}^{2}}{2}+\frac{p_{y}^{2}}{2}+V(x, y ; \gamma, \epsilon)
$$

where, as $\epsilon \rightarrow 0$, the billiard-like potential $V(x, y ; \gamma, \epsilon)$ vanishes in the interior of the scattering billiard domain $D_{\gamma}$ and is of finite height on its boundary (see the next section for exact conditions on $V$ ). The parameter $\epsilon$ controls the steepness of the potential whereas $\gamma$ controls the billiard geometry. We assume that at $\gamma=0$ the billiard has a simple singular periodic orbit - namely, a periodic orbit which is tangent to the billiard boundary at exactly one reflection point. In [7] we proved that in such a setting a linearly stable periodic orbit appears in Eq. (2) for $\epsilon>0$. 
Here, we find that for typical potentials $V$ there exist elliptic islands around these periodic orbits and that their size depends on $\epsilon$ as a power-law - see Theorem 1 and the following discussion.

A natural question that arises here is how prevalent are billiards with simple singular periodic orbits? We argued in [7] that a most natural conjecture would be that the singular periodic orbits may be obtained by an arbitrarily small deformation of the boundary of any dispersing billiard. We also supported this conjecture by some simple numerical simulations. Our results, thus, mean that elliptic islands may appear at a smoothening of any dispersing billiard potential, no matter what the specific geometry of the billiard is. Due to presumed exponential decay of correlation in the dispersing billiards [17], one may expect that the typical period of the elliptic islands for the smoothened billiard-like potential is of order

$$
\sim \lambda^{-1} \ln \varepsilon
$$

where $\lambda$ is the Lyapunov exponent of the (non-smoothened) billiard. Thus, even for extremely steep potentials (very small $\varepsilon$ ) the period of elliptic islands seems to be not so large. In other words, the differences between the statistical behavior of an idealized (billiard) model and its more realistic smooth approximations may become visible on a reasonable time scale.

The proof of Theorem 1 includes the construction of the local return map near the periodic singular orbit, see Eq. (12). This map divides the smooth dispersing billiard-like potentials into universality classes, supplying for each class a computational tool for estimating the size and location of elliptic islands (see Section 5).

The paper is ordered as follows; in Section 2 we formulate the main results of our paper, in Section 3 we construct the local return map near a singular periodic orbit (the proofs are in Appendices A and B), in Section 4 we compute the Birkhoff normal form for the local return map and prove that the first Birkhoff coefficient is not identically zero. In Section 5 we discuss the geometrical interpretation of the universality classes which emerge from the local return map and give more details regarding the visibility and typical period of the islands we have found.

\section{Main result}

Consider a plane scattering (or dispersing) billiard - a billiard in a domain $D \subset R^{2}$ which is a complement to a union of a finite number of strictly convex regions. The boundary of $D$ consists of a finite number of smooth $\left(C^{r+1}, r \geq 2\right)$ curves $S_{1}, S_{2}, \ldots$ joined at the corner points. The angles between the boundary arcs $S_{i}$ at the corner points are non-zero and the curvature of $S_{i}$ is bounded away from zero along the arcs.

The billiard defines a dynamical system (the billiard flow $b_{t}$ ): inertial motion of a pointwise particle inside $D$ and elastic reflections at the boundary - the angle of reflection equals the angle of incidence. We consider a billiard which possesses $a$ (simple) singular periodic orbit.

A basic tool in our analysis is the construction of a regularized billiard to a billiard with a singular periodic orbit. Its construction naturally leads to a new definition of stability or multipliers of the singular orbit. We observe that these multipliers play an essential role in determining the dynamics near the singular periodic orbit. Thus, we define (see Fig. 1):

Definition. Consider the billiard flow in $D$ which has a singular periodic orbit $L$. The domain $D_{L}$ is a regularization of $D$ with respect to $L$ if $L$ is a regular periodic orbit of $D_{L}$, namely $D_{L}$ differs from $D$ by a local smooth deformation of $D$ near the tangencies of $L$ and the support of the deformation is bounded away from any regular reflection point of $L$.

Definition. Consider the billiard flow in $D$ which has a singular periodic orbit $L$. The singular multipliers of $L$ are the multipliers of $L$ in a regularization of $D$ with respect to $L$. 


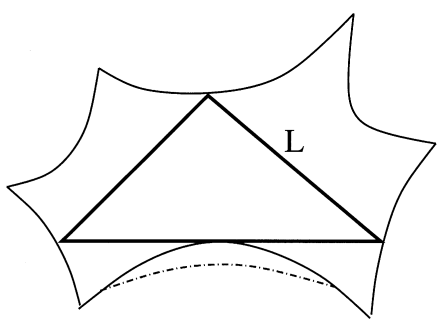

Fig. 1. Domain regularization $\cdot-\cdot-\cdot--\cdot$ is a regularizing deformation of $D$ w.r.t. $L$.

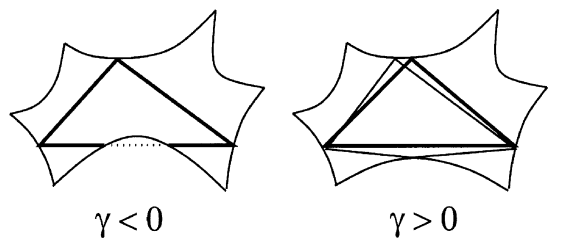

Fig. 2. Domain deformation with $\gamma$.

Notice that while a singular billiard has many regularizing billiards, the singular multipliers of a singular periodic orbit are uniquely defined (because they depend only on the position of the regular reflection points and on the curvature of the boundary there).

\subsection{Smooth billiard potentials}

Consider a smooth Hamiltonian approximation of the given billiard. Namely, consider a two parameter family $h_{t, \epsilon, \gamma}$ of $C^{r}$ Hamiltonian flows associated with

$$
H=\frac{p_{x}^{2}}{2}+\frac{p_{y}^{2}}{2}+V(x, y ; \gamma, \epsilon) \text {. }
$$

We begin with recalling the formulation and the sufficient conditions introduced in [7] which ensure that the Hamiltonian flow of Eq. (3) approximates the billiard flow as $\epsilon \rightarrow 0$ (for a fixed $\gamma$ ). Furthermore, below we describe the required generic dependence on $\gamma$.

The potential $V(x, y ; \gamma, \epsilon)$ tends to zero inside a region $D_{\gamma}$ as $\epsilon \rightarrow 0$ (along with all derivatives, uniformly in any compact subregion of $D_{\gamma}$ ) and it tends to infinity outside $D_{\gamma}$, where $D_{\gamma=0}=D$.

Thus, varying $\gamma$ corresponds to changing the shape of the limiting billiard (see Fig. 2) whereas the parameter $\epsilon$ governs the rate at which the Hamiltonian flow approximates the billiard flow in $D_{\gamma}$.

To have the proper reflection law in the limit, it is necessary that the gradient of the potential stays normal to the boundary of the billiard as $\epsilon \rightarrow 0$. We formalize this requirement as follows:

In a neighborhood of $(\partial D \backslash C)$ ( $C$ is the set of corner points) there exists a pattern function $Q(x, y ; \gamma, \varepsilon)$ whose level lines coincide with the level lines of $V$ in a small neighborhood of the boundary arcs without the corners and which has (along with all derivatives) a finite limit as $\varepsilon \rightarrow 0$ such that at $\gamma=0$ the boundary of the billiard is composed of level lines of $Q(x, y ; 0,0)$ :

$$
\left.Q(x, y ; 0,0)\right|_{(x, y) \in S_{i}} \equiv \text { const. }
$$

Here, we further assume that these level lines depend smoothly on $\gamma$. Thus, at $\gamma \neq 0$ they define a deformed billiard region $D_{\gamma}$ so that the original billiard is now embedded in the one-parameter family of scattering billiards. This is done to unfold the appearance of the singular (tangent) periodic orbit in the original billiard flow. Indeed, 


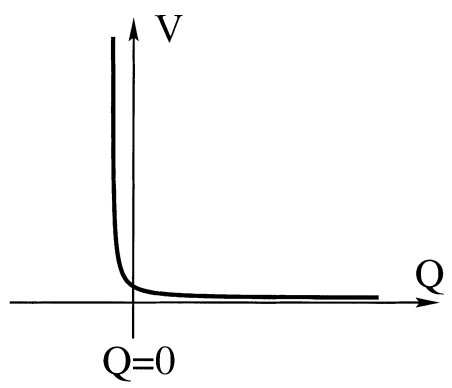

Fig. 3. The form of the barrier function near the boundary.

if the dependence of $D_{\gamma}$ on $\gamma$ is in general position (satisfies Eq. (29)), as for example in Fig. 2, then the tangent periodic orbit disappears, say, at $\gamma<0$ and there is no other periodic orbit in its small neighborhood, whereas at the opposite sign of $\gamma$ two periodic orbits are born, one passing near the former point of tangency without hitting the boundary and the other has a regular reflection close to that point (see [7] for more details).

The existence of the pattern function implies that for each boundary arc $S_{i}$ there exists a barrier function $W_{i}(Q, \epsilon)$ such that near the boundary (in a small neighborhood $N_{i}$ of $S_{i}$ without the corners)

$$
V(x, y ; \epsilon, \gamma)=W_{i}(Q(x, y ; \gamma, \epsilon), \epsilon) .
$$

We assume that $\nabla V$ does not vanish in a finite neighborhood of the boundary arcs, thus:

$$
\left.\nabla Q\right|_{(x, y) \in N_{i}} \neq 0
$$

and

$$
\frac{\mathrm{d}}{\mathrm{d} Q} W_{i}(Q, \varepsilon) \neq 0
$$

Note that the barrier function does not depend explicitly on $(x, y)$ and $\gamma$. It describes the growth of the potential across the boundary arcs and we consider it as afore-hand given and unchanged (though we allow for small $C^{r+1}$ perturbations of the function $Q$ which describes the geometry of the billiard). We consider a large class of possible barrier functions; however, some restrictions are imposed.

First, the barrier function $W_{i}$ must, as $\epsilon \rightarrow 0$, tend to zero at each $Q$ that lies to the inner side of $S_{i}$ and it must tend to infinity on the outer side (Fig. 3). Now note that by Eq. (7) the value of $Q$ may be considered as a function of $W($ and $\varepsilon$ ) near the boundary arc. At small $\varepsilon$, a finite change in $W$ corresponds to a small change in $Q$. Therefore, the following condition makes sense:

As $\varepsilon \rightarrow+0$, for any finite, strictly positive values $V_{1}$ and $V_{2}$, the function $Q(W ; \varepsilon)$ tends to zero uniformly on the interval $V_{1} \leq W \leq V_{2}$ along with all $(r+1)$ derivatives.

Potentials of the above structure (e.g., having the form of Fig. 3 near the boundary) will be called smooth billiard potentials. For example, the following barrier functions give rise to such potentials:

$$
\frac{\epsilon}{Q^{\alpha}}, \quad\left(1-Q^{\alpha}\right)^{1 / \epsilon}, \quad \mathrm{e}^{-Q / \epsilon}, \quad \epsilon|\ln Q|^{\alpha}, \quad \epsilon \ln \ldots|\ln Q|, \quad \alpha>0 .
$$

It is proved in [7] that, as $\epsilon \rightarrow 0$, the Hamiltonian flows (3) with smooth billiard potentials $r$-converge to the billiard flow in $D_{\gamma}$, for any finite $r$. Namely, these converge $C^{r}$ smoothly to the billiard flow outside any neighborhood of singular orbits and $C^{0}$ converge near tangent trajectories. 
2.2. The scaling assumption, asymptotic normal form and elliptic islands

In this paper we impose an additional restriction on the class of smooth billiard potentials which is satisfied by all typical examples (e.g., Eq. (8)), as demonstrated in Section 2.3. Assume that the barrier functions $W(Q, \epsilon)$ satisfy the following scaling assumptions with respect to rescaling of $Q=\delta \tilde{Q}+\beta$ to $\tilde{Q}$ :

[S] For some $\delta=\delta(\epsilon, H)>0, \beta=\beta(\epsilon, H)$ and $v(\epsilon, H)$ such that $\delta \rightarrow 0, \beta \rightarrow 0, \nu / H \rightarrow 0$ as $\epsilon \rightarrow 0$, the function

$$
\tilde{W}_{\epsilon}(\tilde{Q})=\frac{W(\delta \tilde{Q}+\beta, \epsilon)-v}{H \delta^{3 / 2}}
$$

converges as $\epsilon \rightarrow 0$ to a $C^{r+1}$ function $\tilde{W}_{0}(\tilde{Q})$, either for $\tilde{Q}>0$ or for all real $\tilde{Q}$. The convergence is $C^{r+1}-$ uniform on any closed finite interval of values of $\tilde{Q}$ from the domain of definition. Furthermore, the integral

$$
\int_{1}^{+\infty} \tilde{W}_{\epsilon}^{\prime}(q) \frac{\mathrm{d} q}{\sqrt{q}}
$$

converges uniformly, for all sufficiently small $\epsilon$.

In this definition $H$ is the value of energy, see remarks after Theorem 1.

The scaling assumption arises naturally when one requires that the local equations of motion near the tangency will be independent of the small parameter $\epsilon$ and the energy $H$ (see Appendix B, in particular the derivation of Eq. (B.11)). We do not have a clear understanding of a 'physical sense' of the scaling parameters $\delta, \beta, \nu$. It is, however, clear that the scaling assumption would not be satisfied with an arbitrary choice of them. Only those $\delta, \beta$, $v$ for which the scaling assumption is satisfied are good for our purposes. In the examples below, this requirement defines $\delta, \beta, v$ (and the limiting function $\tilde{W}_{0}$ as well) uniquely.

The convergence of the integral (10) allows one to consider a function

$$
F(v)=-\int_{0}^{+\infty} \tilde{W}_{0}^{\prime}\left(v+x^{2}\right) \mathrm{d} x \equiv-\frac{1}{2} \int_{v}^{+\infty} \tilde{W}_{0}^{\prime}(Q) \frac{\mathrm{d} Q}{\sqrt{Q-v}} .
$$

This function arises from integrating the local rescaled equations of motion near the tangency (see Appendix B). It is defined either on $R^{+}$or on $R$ (i.e., on the domain of definition of $\tilde{W}_{0}$ ) and it is $C^{r}$-smooth. Indeed, rewrite

$$
F(v)=-\int_{0}^{1} \tilde{W}_{0}^{\prime}\left(v+x^{2}\right) \mathrm{d} x-\frac{1}{2} \int_{v+1}^{+\infty} \tilde{W}_{0}^{\prime}(Q) \frac{\mathrm{d} Q}{\sqrt{Q-v}} .
$$

The smoothness of both the summands is evident.

We take a convention (see Eqs. (6) and (7)) that the pattern function $Q$ increases across the billiard boundary when moving inwards, therefore, $W^{\prime}$ is negative so $F$ is a positive function.

The following theorem is the main result of the paper:

Theorem 1. Consider a scattering billiard which has a simple singular periodic orbit. Consider a two parameter family of $C^{r}, r \geq 5$, smooth Hamiltonian flows $h_{t}(\epsilon, \gamma)$ of the form(3) with a smooth billiard potential, approximating the billiard flow as $(\epsilon, \gamma) \rightarrow 0$. Assume that the barrier function near the point of tangency satisfies the scaling assumption [S] for some $\delta(\epsilon, H)$ and that the associated function $F$ is such that the range of values of $F^{\prime}(v)$ includes $R^{-}$. Finally, assume that $Q$ dependence on $\gamma$ is in general position (i.e., Eq. (29) holds).

Then, for small $\epsilon$, in the $(\gamma, \epsilon)$ plane there exists a wedge $C^{-} \delta(\epsilon, H)<\gamma<C^{+} \delta(\epsilon, H)$ (with some constant $\left.C^{ \pm}\right)$such that for the parameter values in this wedge, on the energy level $H$ there exist elliptic islands of width proportional to $\delta(\epsilon, H)$. 
Proof. The proof occupies Sections 3 and 4. Roughly speaking, we first show (Proposition 1) that the dynamics of the Hamiltonian flow in an $\mathrm{O}(\delta)$-neighborhood of the tangent periodic trajectory is described, as $\epsilon \rightarrow 0$, by the two-dimensional map

$$
\bar{u}=v, \quad \bar{v}=\xi\left(v+\frac{a}{\sqrt{\kappa}} F(v)\right)-(\xi-2) \Gamma-u .
$$

It is the limit of the two-dimensional return map of the Hamiltonian flow to a local cross-section near the tangent trajectory, written in some rescaled coordinates. $\kappa$ is the billiard's curvature at the tangency and the quantities $\xi$ and $a(|\xi|>2, a>0)$ are defined by the billiard flow at $\epsilon=0, \gamma=0$; In fact, $\xi$ is just the sum of the singular multipliers of $L ; \Gamma$ is the rescaled parameter $\gamma$.

We call Eq. (12) the asymptotic normal form of the local Poincare map near the tangent trajectory. In Proposition (31), we show that the map (12) has an elliptic island of finite size (in the rescaled to $\delta$ variables $u$ and $v$ ) for a finite range of values of $\Gamma$. Returning to the original, non-scaled variables gives the $\delta$-size island which appears in the wedge as stated.

Few remarks are now in order; first, the theorem implies that given a billiard with a simple singular periodic orbit there exist one parameter family of Hamiltonian flows $h_{t}(\epsilon, \gamma(\epsilon))$ which $r$-converge to the billiard flow and for which elliptic islands of size $\delta(\epsilon, H)$ exist for all $\epsilon<\epsilon_{0}$.

Second, the size of the island depends, of course, on the choice of the coordinates - we choose the most natural coordinates so the theorem applies directly to the islands size as observed in the phase space coordinates (3). To be specific, given the singular periodic orbit $L$, we choose the origin as the point of tangency, and the $x$-axis parallel to $L$ at that point. A local Poincaré map in a neighborhood of $L$ is defined by taking $x=$ const, and the coordinates on the cross-section are the variables $y$ and $y^{\prime}=p_{y} / p_{x}$ (here $p_{x} \approx \sqrt{H}$, hence, when large $H$ values are considered this additional factor arises).

Thirdly, the billiard flow is clearly independent of $H$, hence, using assumption [S] it is possible to obtain local return map which is independent of $H$. It follows that for the Hamiltonian flow islands appear for all $H$ values (and their size depends on $H$ via $\delta(\epsilon, H)$ and $\sqrt{H}$ as explained above). For greater genericity, we also allow the chosen value of $H$ to depend on $\epsilon$.

Finally, the scaling assumption $[\mathrm{S}]$ is satisfied by all potentials listed in Eq. (8). Moreover, given a barrier function $W$, in many cases, the scaling constant $\delta$ and the function $F$ involved in the above estimate of the size of elliptic islands are fairly easy to compute. Before embarking into the details of the proof, we demonstrate the above statement for typical examples of the list (8).

\subsection{Islands size for specific potentials}

Consider the barrier function

$$
W(Q, \epsilon)=\frac{\epsilon}{Q^{\alpha}} .
$$

It satisfies assumption [S] provided

$$
\delta(\epsilon, H)=\left(\frac{\epsilon}{H}\right)^{1 /(\alpha+3 / 2)}, \quad v=0, \quad \beta=0,
$$

for which

$$
\tilde{W}_{0}(\tilde{Q})=\frac{1}{\tilde{Q}^{\alpha}} .
$$


Integration of Eq. (11) gives in this case

$$
F(v)=\frac{\sqrt{\pi}}{2} \frac{\Gamma(\alpha+1 / 2)}{\Gamma(\alpha)} \frac{1}{v^{\alpha+1 / 2}} .
$$

The function $F$ satisfies the condition of the theorem so for such potentials the size of elliptic islands is given by $\delta$ of Eq. (13), namely it is polynomial in $\epsilon / H$. Note that the size of islands is larger (asymptotically) for larger $\alpha$ (though the limit $\alpha \rightarrow \infty$ cannot be taken in Eq. (13)).

Choosing

$$
W(Q, \epsilon)=\exp (-Q / \epsilon)
$$

will satisfy the scaling assumption, provided

$$
\delta=\epsilon, \quad v=0, \quad \beta=-\epsilon \ln \left(\delta^{3 / 2} H\right),
$$

for which

$$
\tilde{W}_{0}(\tilde{Q})=\exp (-\tilde{Q})
$$

and

$$
F(v)=\frac{\sqrt{\pi}}{2} \mathrm{e}^{-v}
$$

Thus, in this case the elliptic islands are of size $\epsilon$, independent asymptotically of the value of $H$ (in the ( $\left.y, y^{\prime}\right)$ coordinates).

An example which has been used by many authors $[18,19]$ is:

$$
W(Q, \epsilon)=\left(1-Q^{\alpha}\right)^{1 / \epsilon}
$$

It is obviously equivalent to

$$
W(Q, \epsilon)=\exp \left(-Q^{\alpha} / \epsilon\right)
$$

which is analogous to the previous example; if $\epsilon^{3 / 2 \alpha} H \rightarrow 0$, one may assume

$$
\delta=\frac{\epsilon^{1 / \alpha}}{\alpha}\left(-\ln \left(\epsilon^{3 / 2 \alpha} H\right)\right)^{(1-\alpha) / \alpha}, \quad v=0, \quad \beta=\left(-\epsilon \ln \left(\delta^{3 / 2} H\right)\right)^{1 / \alpha} .
$$

Here again

$$
\tilde{W}_{0}(\tilde{Q})=\exp (-\tilde{Q})
$$

and

$$
F(v)=\frac{\sqrt{\pi}}{2} \mathrm{e}^{-v}
$$

Thus, the islands size is again polynomial in $\epsilon$. The critical exponent of $H \propto \epsilon^{-3 /(2 \alpha)}$ arises from the analysis if $H$ grows faster - different asymptotics applies. Also, notice the different behavior for $\alpha>1$ versus $\alpha<1$, again we see that steeper decay of the potential gives rise to larger islands. 


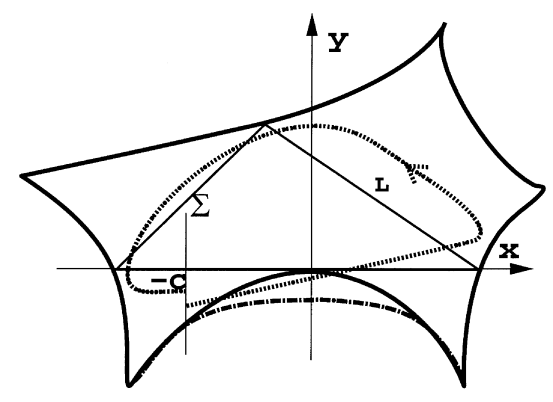

Fig. 4. Auxiliary regular local map near tangency. $\cdot-\cdot-\cdot-\cdot \cdot$ is the regularization of $D$ w.r.t. $L$ and $\ldots$ is a trajectory of the auxiliary Hamiltonian flow.

Finally, for

$$
W(Q, \epsilon)=\epsilon|\ln Q|^{\alpha}
$$

take

$$
\delta=\left(\frac{\alpha \epsilon}{H}\right)^{2 / 3}\left(\frac{2}{3}\left|\log \frac{\alpha \epsilon}{H}\right|\right)^{2(\alpha-1) / 3}, \quad v=\epsilon|\ln \delta|^{\alpha}, \quad \beta=0,
$$

for which

$$
\tilde{W}_{0}(\tilde{Q})=-\ln \tilde{Q}
$$

and

$$
F(v)=\frac{\pi}{2 \sqrt{v}} .
$$

\section{The Poincaré map near a tangent periodic orbit}

Here, we formulate and prove Proposition 1 describing the local return map near a simple singular periodic orbit. Consider the two parameter family of Hamiltonian systems (3) with smooth billiard potentials obeying the scaling assumption [S] (by which $\delta, \beta, \tilde{W}(\tilde{Q})$ are determined), which $r$-converge to the embedding family of the billiard flow with the simple singular periodic orbit $L$ as above.

Choose the coordinates $(x, y)$ such that the origin is at the point of tangency and the $x$-axis is tangent to the boundary - i.e., it is parallel to the tangent piece of the singular trajectory $L$. On a fixed energy level $H$ take a small two-dimensional cross section $\Sigma=\{x=-c\}$ to this piece of $L$, where $c$ is some fixed small positive number (see Fig. 4). For greater genericity, we assume that $c$ may depend on $\epsilon$ and $\gamma$ and it tends to some small positive value as $\epsilon, \gamma \rightarrow 0$.

The variables $y$ and $y^{\prime} \equiv p_{y} / p_{x}$ serve as the coordinates on $\Sigma$ : given fixed $y^{\prime}$, the value of $p_{x}$ is found from the energy constrain because $\partial H / \partial p_{x} \equiv p_{x}\left(1+y^{\prime 2}\right)$ does not vanish on $\Sigma$ due to the choice of the coordinate frame.

Proposition 1. There is a region on the cross-section $\Sigma$ on the energy level $H$ where the local return map $B_{\epsilon}$ of the smooth Hamiltonian flow may be reduced, by an affine transformation $\left(y, y^{\prime}\right) \mapsto(u, v)$ which has a finite limit (with non-zero Jacobian) as $\epsilon \rightarrow 0$, to the form:

$$
\left\{\begin{array}{l}
\bar{u}=v+\cdots \\
\bar{v}=\xi\left(v+\frac{a}{\sqrt{\kappa}} \delta F\left(\frac{v+\cdots}{\delta}\right)\right)-(\xi-2) v_{\gamma}-u+\cdots
\end{array}\right.
$$


where the function $F$ is defined by Eq. (11), $\delta(\epsilon, H)$ is the scaling constant from Eq. (9), $\kappa$ is the curvature of the billiard at the tangency point at $\gamma=0$, the quantities $a$, $\xi$ and $v_{\gamma}$ have a finite limit as $\epsilon \rightarrow 0$ where $|\xi|>2, a>0$, and $v_{\gamma}$ vanishes at $\gamma=0, \epsilon=0$. The map (17) is defined at $\|(u, v)\| \leq K \delta$ ( $v$ must be strictly positive when the domain of definition of $F$ is $R^{+}$) where $K$ may be taken arbitrarily large provided $\epsilon$ is small enough. The dots in Eq. (17) stand for smooth functions of $(u / \delta, v / \delta)$ which, along with all derivatives, are $\mathrm{o}(\delta)$ as $\epsilon \rightarrow 0$.

Proof. Similar to [7], we construct the return map $B_{\epsilon}$ from two artificial maps $B_{\epsilon}^{(r)}$ and $B_{\epsilon}^{(s)}$, both defined as the maps of the cross-section $\Sigma$ to itself. In Sections 3.1 and 3.2 we describe these constructions (the details of the corresponding proofs are deferred to Appendices A and B) and in Section 3.3 we prove that their composition may be transformed to Eq. (17).

\subsection{The regular map}

The regular map $B_{\epsilon}^{(r)}: \Sigma \rightarrow \Sigma$ is constructed in Appendix A by ignoring the passage near the tangent point at the origin. Namely, we locally modify the function $Q$ near the point of tangency so that the billiard's boundary $Q(x, y ; \gamma, 0)=0$ is moved outward, see Fig 3. Given a $c$, the modified $Q$ is changed at most for $|x|<c$, near $Q=0$. For this regularized billiard, the periodic orbit $L$ survives and becomes non-singular. We consider an auxiliary Hamiltonian flow which corresponds to the modified function $Q$ in Eqs. (3) and (5). The regular map $B_{\epsilon}^{(r)}$ is the Poincaré map for the auxiliary Hamiltonian flow. Since the auxiliary Hamiltonian flow is still generated by a smooth billiard potential, it $r$-converges [7] to the corresponding regularized billiard flow. In particular, it $C^{r}$-converges to the regularized billiard flow near the now non-singular periodic orbit $L$. Thus, $B_{\epsilon}^{(r)}$ is a $C^{r}$-diffeomorphism which, as $\epsilon \rightarrow 0$, approaches a finite limit, namely, the Poincaré map $B_{0}^{(r)}$ of the regularized billiard flow near $L$. Thus, using the hyperbolic structure of the regularized billiard map $B_{0}^{(r)}$, we prove in Appendix A the following:

Lemma 1. The map $B_{\epsilon}^{(r)}: \Sigma \rightarrow \Sigma$ is given by:

$$
B_{\epsilon}^{(r)}\left(\begin{array}{l}
y \\
y^{\prime}
\end{array}\right)=\left(\begin{array}{l}
y_{\gamma, \epsilon} \\
p_{\gamma, \epsilon}
\end{array}\right)+\left(\begin{array}{ll}
b_{11} & b_{12} \\
b_{21} & b_{22}
\end{array}\right)\left(\begin{array}{l}
y-y_{\gamma, \epsilon} \\
y^{\prime}-p_{\gamma, \epsilon}
\end{array}\right)+\cdots
$$

where $\left(y_{\gamma, \epsilon}, p_{\gamma, \epsilon}\right)$ are the coordinates of the hyperbolic fixed point of $B_{\epsilon}^{(r)}$ corresponding to the periodic orbit $L$ at $(\epsilon=0, \gamma=0)$ and the dots stand for nonlinear terms. Then, $y_{\gamma, \epsilon}, p_{\gamma, \epsilon}$ have a finite limit as $\epsilon \rightarrow 0$ with $y_{0,0}=p_{0,0}=0$, and the linearization matrix $\mathcal{B}=\left\{b_{i j}(\gamma, \epsilon)\right\}$ satisfies the following:

- The sign of all the coefficients $b_{i j}$ is identical:

$$
\operatorname{sign} b_{i j}=\operatorname{sign} \mathcal{B} .
$$

- Moreover,

$$
\operatorname{sign}\left(b_{12}-c b_{11}\right)=\operatorname{sign}\left(b_{22}-c b_{21}\right)=\operatorname{sign} \mathcal{B} .
$$

- The determinant of $\mathcal{B}$ is 1 .

- The magnitude of the trace of $\mathcal{B}$ is larger than 2.

\subsection{The singular map}

In Appendix B the singular part $B_{\epsilon}^{(s)}: \Sigma \rightarrow \Sigma$ is constructed; define the cross-section $\Sigma^{\prime}=\{x=c\}$. Recall that the auxiliary Hamiltonian flow coincides with the original flow outside the size $c$ neighborhood of the point 


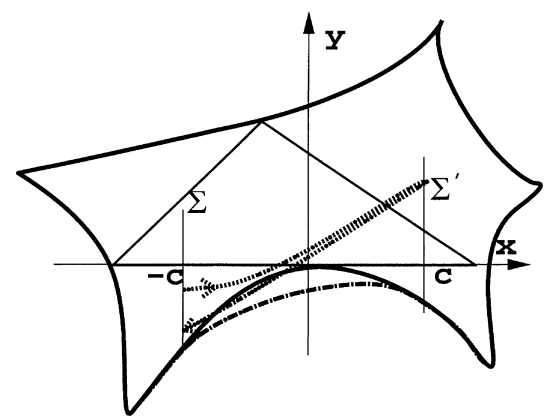

Fig. 5. Singular local map near tangency. $\cdot-\cdot-\cdot \cdot-\cdot$ is the regularization of $D$ w.r.t. $L$ and $\ldots$ is a trajectory of the singular local map.

of tangency. Now, define $B_{\epsilon}^{(s)}$ as the composition of the map $\Sigma \rightarrow \Sigma^{\prime}$ by the orbits of the original Hamiltonian flow and, then, the map $\Sigma^{\prime} \rightarrow \Sigma$ defined by the backward orbits of the auxiliary Hamiltonian flow, see Fig. 5. By construction, the local Poincaré map $B_{\epsilon}$ is the superposition

$$
B_{\epsilon}=B_{\epsilon}^{(r)} \circ B_{\epsilon}^{(s)} .
$$

The singular part is computed locally, using the scaling property. More precisely, in the appendix we prove the following:

Lemma 2. The map $B_{\epsilon}^{(s)}: \Sigma \rightarrow \Sigma$ is given by:

$$
\begin{aligned}
& \bar{y}=y_{0}(\epsilon)+y-c \frac{\delta}{\sqrt{\kappa}} F\left(\frac{y+c y^{\prime}+o(\delta)}{\delta}\right)+o(\delta) \\
& \bar{y}^{\prime}=p_{0}(\epsilon)+y^{\prime}+\frac{\delta}{\sqrt{\kappa}} F\left(\frac{y+c y^{\prime}+o(\delta)}{\delta}\right)+o(\delta),
\end{aligned}
$$

where $y^{\prime}=p_{y} / p_{x}, F$ is defined by Eq. (11), and $y_{0}, p_{0} \rightarrow 0$ as $\epsilon \rightarrow 0$; the quantity $\kappa$ is half the curvature at the point of tangency. The formula (22) is valid for $\left\|\left(y / \delta, y^{\prime} / \delta\right)\right\| \leq K$ where $K$ may be taken arbitrarily large, provided $\epsilon$ is small enough; also, if $F$ is defined only on $R^{+}$, the allowed values of $y$ and $y^{\prime}$ are such that $\left(y+c y^{\prime}\right) / \delta$ is strictly positive. The symbol o $(\delta)$ denotes functions of $\left(y / \delta, y^{\prime} / \delta\right)$ which, along with all derivatives with respect to $\left(y / \delta, y^{\prime} / \delta\right)$, decay to zero faster than $\delta(\epsilon)$ as $\epsilon \rightarrow 0$.

\subsection{The local return map}

It follows from our construction (Lemmas 1 and 2) that the composition of the singular flow and the regularized billiard gives the desired return map (see Eq. (21)).

Now, superposition of $B_{\epsilon}^{(r)}$ (see Eq. (18)) and $B_{\epsilon}^{(s)}$ (see Eq. (22)) gives the following formula for the Poincaré map $B_{\epsilon}$ :

$$
\left(\begin{array}{c}
\bar{y} \\
\bar{y}^{\prime}
\end{array}\right)=\left(\begin{array}{c}
y_{\gamma, \epsilon} \\
p_{\gamma, \epsilon}
\end{array}\right)+\mathcal{B}\left(\begin{array}{c}
y-y_{\gamma, \epsilon}+y_{0} \\
y^{\prime}-p_{\gamma, \epsilon}+p_{0}
\end{array}\right)+\mathcal{B}\left(\begin{array}{c}
-c \\
1
\end{array}\right) \frac{\delta}{\sqrt{\kappa}} F\left(\frac{y+c y^{\prime}+o(\delta)}{\delta}\right)+o(\delta)
$$

where

$$
\mathcal{B}=\left(\begin{array}{ll}
b_{11} & b_{12} \\
b_{21} & b_{22}
\end{array}\right)
$$


Let us make the affine transformation

$$
v=y+c y^{\prime}, \quad u=(1, c) \mathcal{B}^{-1}\left(\begin{array}{c}
y-y_{\gamma, \epsilon} \\
y^{\prime}-p_{\gamma, \epsilon}
\end{array}\right)+(1, c)\left(\begin{array}{c}
y_{\gamma, \epsilon}-y_{0} \\
p_{\gamma, \epsilon}-p_{0}
\end{array}\right)
$$

recall that $\operatorname{det} \mathcal{B}=1$, hence

$$
\mathcal{B}^{-1}=\left(\begin{array}{rr}
b_{22} & -b_{12} \\
-b_{21} & b_{11}
\end{array}\right) \text {. }
$$

It is easy to see that this change of coordinates brings the map (23) to the form (17), where

$$
\begin{aligned}
\xi & =\operatorname{tr} \mathcal{B}, \\
a & =\frac{(1, c) \mathcal{B}(-c, 1)^{\mathrm{T}}}{\xi}
\end{aligned}
$$

and

$$
v_{\gamma}=y_{\gamma, \epsilon}+c p_{\gamma, \epsilon}-\frac{\left(b_{11}+b_{21} c-1\right) y_{0}+\left(b_{12}+b_{22} c-c\right) p_{0}}{\xi-2} .
$$

Moreover, $a>0$ by Eq. (20) and $|\xi|>2$ by Eq. (25) (see Lemma 1).

Finally, the determinant of the transformation is given by:

$$
J=\frac{\partial(u, v)}{\partial\left(y, y^{\prime}\right)}=(1, c) \mathcal{B}(-c, 1)^{\mathrm{T}}=\xi a,
$$

hence it clearly has a finite, non-vanishing limit as $\epsilon \rightarrow 0$, completing the proof of Proposition 1.

\subsection{Non-degenerate dependence on $\gamma$}

Here, we establish that under the assumptions of Proposition 1, and if additionally, the dependence of the smooth billiard potential on $\gamma$ is in general position, then $v_{\gamma}$ of Eq. (17) can be replaced by $\gamma$.

Indeed, consider the billiard flow $(\epsilon=0)$; then from Eq. (27) $v_{\gamma}=2\left(y_{\gamma, \epsilon=0}+c p_{\gamma, \epsilon=0}\right)$ where the point $M_{\gamma}=\left(y_{\gamma 0}, p_{\gamma 0}\right)$ is the hyperbolic fixed point of the regular map which corresponds to the regularized billiard. Notice that on the cross-section $\Sigma:\{x=-c\}$ the straight line $v=y+c y^{\prime}=0$ is tangent to the singularity line (the curve of initial conditions corresponding to tangent trajectories). Moreover, the point $M_{0}$ belongs to the singularity line by assumption, and by definition $M_{0}=(0,0)$. Thus, $v_{0}=0$. We make the following non-degeneracy assumption:

The fixed point $M_{\gamma}$ of the regular map $B_{0}^{(r)}$ crosses the singularity line with non-zero velocity as $\gamma$ varies.

Since $v$ measures the distance from the singularity line, it is equivalent to

$$
\frac{\partial v_{\gamma}}{\partial \gamma} \neq 0
$$

Thus $\gamma$ may be rescaled so that

$$
v_{\gamma} \equiv \gamma
$$

Notice that the non-degeneracy assumption is formulated in terms of the billiard flow solely; thus, it describes how the boundary of the billiard depends on $\gamma$. It is a condition of general position: if it is not satisfied, it may be easily achieved by a small smooth perturbation of the function $Q(x, y ; \gamma, 0)$ : the position of the singularity line is 
determined only by the local behavior of $Q$ near the point of tangency whereas the position of the auxiliary fixed point $M$ is determined only by the local behavior of $Q$ at the points of regular collision of $L$ with the boundary.

The non-degeneracy condition is simple and natural when the dependence on $\gamma$ is localized near the tangent point (e.g., when artificially embedding the singular billiard in a family of billiards as in regularization); in this case the periodic orbit of the auxiliary billiard does not move as $\gamma$ varies. This means that $y_{\gamma}^{\prime}=0$. Since the coordinates on $\Sigma$ are defined so that the orbit starting at $\left(y=0, y^{\prime}=0\right)$ is tangent to the boundary, it follows that $\partial y_{\gamma} / \partial \gamma \neq 0$ provided the boundary near the point of tangency moves, as $\gamma$ varies, with non-zero velocity in the normal direction (e.g., see Fig 4).

\section{Islands}

To complete the proof of Theorem 1, we now establish that the Poincare map found in Proposition 1 attains elliptic islands as stated in the Theorem. Let us consider the following rescaling of Eq. (17):

$$
u^{\prime}=\frac{u}{\delta}, \quad v^{\prime}=\frac{v}{\delta}, \quad \Gamma=\frac{v_{\gamma}}{\delta} .
$$

In the new variables the map (17) has the map (12) as a limit as $\epsilon \rightarrow 0$. Since $\mathrm{O}(1)$ intervals in the rescaled variables correspond to $\mathrm{O}(\delta)$ intervals in the original variables, it follows that once we show that for a finite range of values of $\Gamma$ the map (12) has an elliptic island of a finite size, we are done. The idea is to use KAM-theory in order to establish the existence of the island around an elliptic periodic point, like in $[8,20,21]$ and in many other papers.

Thus, Theorem 1 reduces to the following statement.

Proposition 2. Assume the range of values of $F^{\prime}(v)$ includes $R^{-}$. Then, for any $|\xi|>2$ and $a>0$ there exists an interval of values of $\Gamma$ for which the map (12) has an elliptic fixed point with non-zero first coefficient in the Birkhoff normal form.

Proof. Fixed points of Eq. (12) are:

$$
u_{f}=v_{f}, \quad \Gamma_{f}\left(v_{f}\right)=v_{f}+\frac{a \xi}{\sqrt{\kappa}(\xi-2)} F\left(v_{f}\right)
$$

which defines $\Gamma_{f}\left(v_{f}\right)$ for all $v_{f}$ in the domain of definition of $F\left(\right.$ e.g., $\left.R^{+}\right)$. The fixed points are linearly stable if and only if:

$$
v_{f} \in I_{\mathrm{S}}=\left\{v_{f} \mid-\left(1+\frac{2}{|\xi|}\right)<\frac{a}{\sqrt{\kappa}} F^{\prime}\left(v_{f}\right)<-\left(1-\frac{2}{|\xi|}\right)\right\}
$$

Namely $I_{\mathrm{S}}$ is a union of open intervals of stability in $v_{f}$, which is non-empty if, for example, the range of $F^{\prime}(v)$ includes $R^{-}$as required, leading to intervals of the corresponding $\Gamma_{f}$ values.

On $I_{\mathrm{s}}$, the eigenvalues of the fixed point are $\mathrm{e}^{\mathrm{ti} \omega}$ where

$$
\cos \omega=\frac{\xi}{2}\left(1+\frac{a}{\sqrt{\kappa}} F^{\prime}\left(v_{f}\right)\right) .
$$

Since $F^{\prime}$ takes all negative values, there exists at least one interval of linear stability from $I_{\mathrm{s}}$ on which the value of $\omega$ runs from 0 to $\pi$.

To prove the lemma we need to show that at least for one $v_{f}$ from the given interval the first coefficient of the Birkhoff normal form at the elliptic fixed point is non-zero (according to Moser theorem this will prove that at the corresponding value of $\Gamma$ and for all close $\Gamma$, there exists an elliptic island surrounding the fixed point). 
The sought Birkhoff coefficient equals to

$$
B\left(v_{f}\right)=-\frac{1}{8 \sin ^{3} \omega}\left(\frac{\xi a}{2 \sqrt{\kappa}} F^{\prime \prime \prime}\left(v_{f}\right)+\left(\frac{\xi a}{2 \sqrt{\kappa}} F^{\prime \prime}\left(v_{f}\right)\right)^{2} \frac{(1+4 \cos \omega)}{(1-\cos \omega)(1+2 \cos \omega)}\right) .
$$

Indeed, the linear transformation $z=\mathrm{e}^{\mathrm{i} \omega}\left(v-v_{f}\right)-\left(u-u_{f}\right)$ brings the map (12) to the following form near the fixed point:

$$
\bar{z}=\mathrm{e}^{\mathrm{i} \omega} z-\frac{\xi a}{\sqrt{\kappa}} F^{\prime \prime}\left(v_{f}\right) \frac{\mathrm{e}^{\mathrm{i} \omega}}{8 \sin ^{2} \omega}\left(z-z^{*}\right)^{2}+\mathrm{i} \frac{\xi a}{\sqrt{\kappa}} F^{\prime \prime \prime}\left(v_{f}\right) \frac{e^{\mathrm{i} \omega}}{48 \sin ^{3} \omega}\left(z-z^{*}\right)^{3}+\cdots
$$

where the dots stand for higher order terms.

An arbitrary two-dimensional conservative map near an elliptic fixed point is written as

$$
\bar{z}=\mathrm{e}^{\mathrm{i} \omega} z+\mathrm{e}^{\mathrm{i} \omega}\left(\alpha z^{2}-2 \alpha^{*} z z^{*}+\eta z^{* 2}\right)+\mathrm{e}^{\mathrm{i} \omega} \beta z^{2} z^{*}+\cdots
$$

where

$$
\operatorname{Re} \beta=\alpha \alpha^{*}-\eta \eta^{*}
$$

(which is necessary to have the Jacobian equal to one) and the dots stand for the rest of cubic and higher order terms. In the case of map (36)

$$
\alpha=\eta=-\frac{\xi a F^{\prime \prime}\left(v_{f}\right)}{8 \sqrt{\kappa} \sin ^{2} \omega}
$$

and

$$
\beta=-\mathrm{i} \frac{\xi a F^{\prime \prime \prime}\left(v_{f}\right)}{16 \sqrt{\kappa} \sin ^{3} \omega} .
$$

If $\omega \neq 2 \pi / 3$, a simplectic transformation of the form

$$
z^{\prime}=z-\left[\frac{\mathrm{e}^{\mathrm{i} \omega} \alpha}{\mathrm{e}^{2 \mathrm{i} \omega}-\mathrm{e}^{\mathrm{i} \omega}} z^{2}-2 \frac{\mathrm{e}^{\mathrm{i} \omega} \alpha^{*}}{1-\mathrm{e}^{\mathrm{i} \omega}} z z^{*}+\frac{\mathrm{e}^{\mathrm{i} \omega} \eta}{\mathrm{e}^{-2 \mathrm{i} \omega}-\mathrm{e}^{\mathrm{i} \omega}} z^{* 2}\right]+\left[\frac{(1 / 2) \eta \eta^{*}}{1-\cos 3 \omega}-\frac{(1 / 2) \alpha \alpha^{*}}{1-\cos \omega}\right] z^{2} z^{*}+\cdots
$$

eliminates all quadratic terms in Eq. (37) and brings it to the form (dropping ')

$$
\bar{z}=\mathrm{e}^{\mathrm{i} \omega} z+\mathrm{ie}^{\mathrm{i} \omega} B z^{2} z^{*}+\cdots
$$

where $B$ is the first Birkhoff coefficient ${ }^{2}$ :

$$
B=\operatorname{Im} \beta+\frac{\sin \omega}{1-\cos \omega}\left[\eta \eta^{*} \frac{1-2 \cos \omega}{1+2 \cos \omega}-3 \alpha \alpha^{*}\right] .
$$

Plugging Eq. (39) and (40) in Eq. (43) gives Eq. (35).

If $\omega \neq 2 \pi / 3, \omega \neq \pi / 2$, all the other cubic terms in Eq. (42) are non-resonant, hence, they can be eliminated by the further normalizing transformation. Then, in the simplectic polar coordinates $z=\sqrt{r} \mathrm{e}^{\mathrm{i} \theta}$ the map becomes

$$
\bar{r}=r+o\left(r^{2}\right), \quad \bar{\theta}=\theta+\omega+B r+o(r) .
$$

When $\left.\bar{\theta}_{r=0}^{\prime}\right|_{r=B} \equiv 0$, the KAM-theory is applied to this map at small $r$, which gives the existence of the sought elliptic island.

\footnotetext{
${ }^{2}$ At $\beta=0$ this expression coincides with that found in [20].
} 
Now, note that the quantity $B\left(v_{f}\right)$ given by Eq. (35) cannot be identically zero on the interval of values of $v_{f}$ under consideration. Indeed, if for example $F^{\prime \prime}\left(v_{f}\right)$ never vanishes, then $B\left(v_{f}\right)$ tends to infinity when approaching the bifurcation point $v_{f}=v_{f}^{1}$ which corresponds to $\cos \omega=1$. One can check that even if $F^{\prime \prime}\left(v_{f}^{1}\right)$ vanishes but the zero is of finite order, then still $B\left(v_{f}\right)$ is not identically zero near $v_{f}=v_{f}^{1}$. Indeed, in this case, for some finite $n \geq 1$ and $d \neq 0$,

$$
\begin{aligned}
F^{\prime}\left(v_{f}\right) & =F^{\prime}\left(v_{f}^{1}\right)+d\left(v_{f}-v_{f}^{1}\right)^{n}+\cdots, \\
F^{\prime \prime}\left(v_{f}\right) & =n d\left(v_{f}-v_{f}^{1}\right)^{n-1}+\cdots, \\
F^{\prime \prime \prime}\left(v_{f}\right) & =n(n-1) d\left(v_{f}-v_{f}^{1}\right)^{n-2}+\cdots,
\end{aligned}
$$

then, from Eq. (34)

$$
\cos \omega=1+\frac{d a \xi}{2 \sqrt{\kappa}}\left(v_{f}-v_{f}^{1}\right)^{n}+\cdots
$$

hence, from Eq. (35)

$$
B\left(v_{f}\right)=\frac{d a \xi}{16 \sqrt{\kappa} \sin ^{3} \omega} n\left(1+\frac{8}{3} n\right)\left(v_{f}-v_{f}^{1}\right)^{n-2}+\cdots \not \equiv 0
$$

This proves the lemma for the case where the function $F$ is analytic (as in all our examples). In this case, it follows that $B$ may vanish only at isolated points in the interval of stability.

Without the assumption of the analyticity, if $B\left(v_{f}\right)$ were identically zero on the interval under consideration, we would have by Eq. (35)

$$
\frac{\xi a}{2 \sqrt{\kappa}} F^{\prime \prime \prime}\left(v_{f}\right)+\left(\frac{\xi a}{2 \sqrt{\kappa}} F^{\prime \prime}\left(v_{f}\right)\right)^{2} \frac{(1+4 \cos \omega)}{(1-\cos \omega)(1+2 \cos \omega)}=0
$$

or, in view of Eq. (34),

$$
\frac{\mathrm{d}^{2} \cos \omega}{\mathrm{d} v_{f}^{2}}+\left(\frac{\mathrm{d} \cos \omega}{\mathrm{d} v_{f}}\right)^{2} \frac{(1+4 \cos \omega)}{(1-\cos \omega)(1+2 \cos \omega)}=0 .
$$

This equation is easily integrated; on the interval $\cos \omega \in[-1 / 2,1]$ we get

$$
\frac{\mathrm{d} \cos \omega}{\mathrm{d} v_{f}}=c \sqrt[3]{(1-\cos \omega)^{5}(1+2 \cos \omega)},
$$

where $c$ is some constant which cannot be zero because $\cos \omega$ must run all the interval under consideration as $v_{f}$ varies. At $\cos \omega$ close to 1 we have

$$
\frac{\mathrm{d} \cos \omega}{\mathrm{d} v_{f}} \sim(1-\cos \omega)^{5 / 3} .
$$

This means that $\omega$ never approaches zero at finite $v_{f}$ which contradicts the initial assumption (that the range of $F^{\prime}$ includes all negative values, see Eq. (34)).

As an example, consider numerical simulations of the map (12) for $W(Q)=\epsilon / Q$ which confirm the existence of $\mathrm{O}(1)$ islands; the map (12) becomes:

$$
\left\{\begin{array}{l}
\bar{u}=v \\
\bar{v}=\xi v-(\xi-2) \Gamma-u+\xi \frac{\pi}{4} \frac{a}{\sqrt{\kappa}} \frac{1}{v^{3 / 2}}
\end{array}\right.
$$



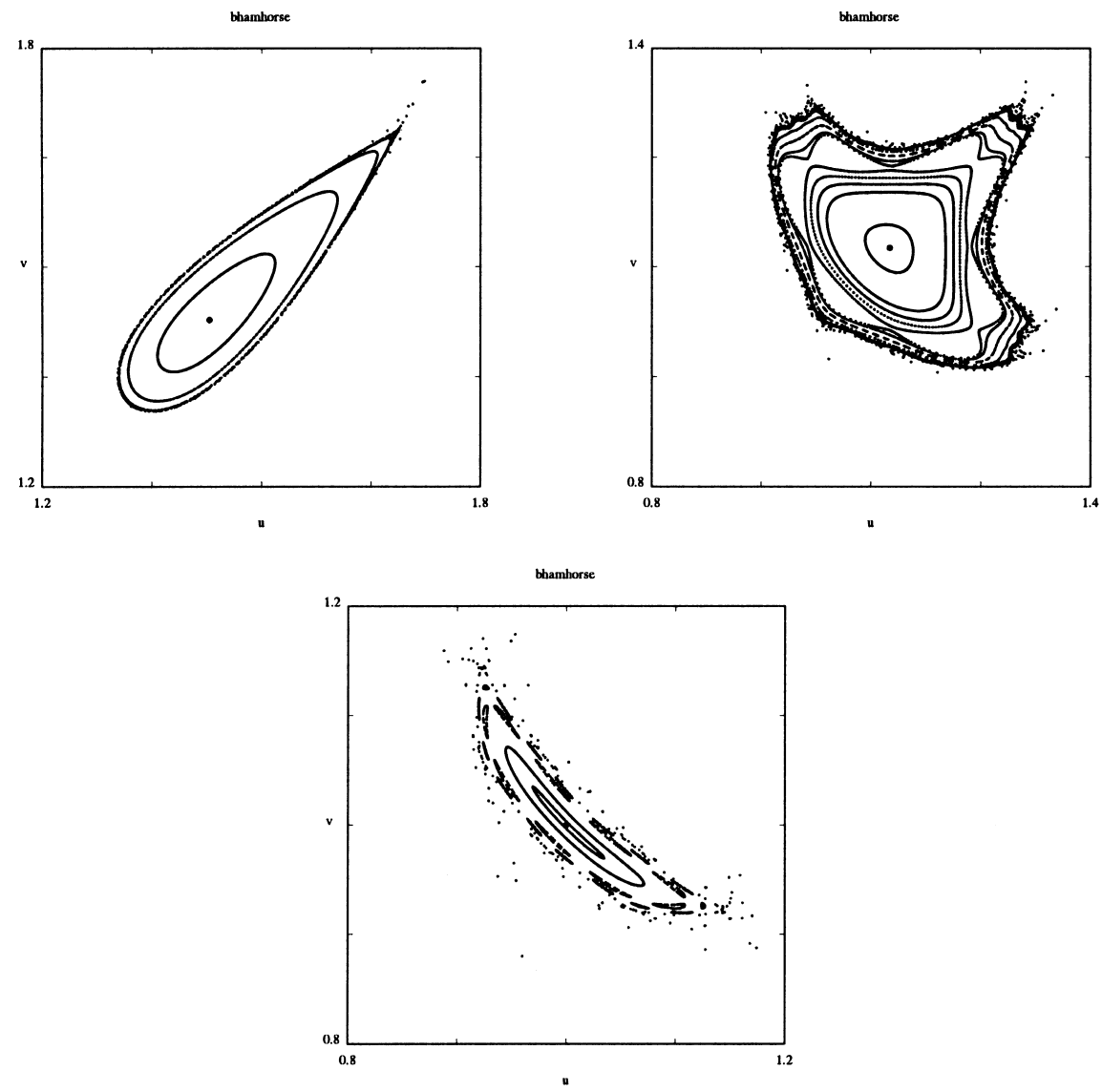

Fig. 6. Rescaled local map near singular periodic orbit. Orbits of Eq. (52) $(W(Q)=\epsilon / Q, \xi=4, \pi / 4 a / \sqrt{\kappa}=1)$ (a) $\Gamma=2.6$, (b) $\Gamma=2.8$, (c) $\Gamma=3$

Taking $\xi=4,(\pi / 4)(a / \sqrt{\kappa})=1$ makes $I_{\mathrm{s}}=\left(1,3^{0.4} \approx 1.5518\right)$ and thus an interval of stability for $\Gamma \in$ $\left((5 / 3) 3^{0.4} \approx 2.586,3\right.$. $)$, Fig. 6 confirms the existence of elliptic island for $\Gamma=2.6,2.8$. In fact it is seen that even for $\Gamma=3$. the stability island still exists.

\section{Discussion}

Our main result, Theorem 1, together with assumption [S], supplies a computational tool for estimating the size of elliptic islands for specific potentials. For natural Hamiltonians which appear in physical setting, the estimated size of the islands is polynomial in $\epsilon$, where $\epsilon$ is the small parameter which controls the steepness of the potential near the core. Moreover, the dependence of the islands size on the energy has also been explicitly computed (see Sections 2.2 and 2.3) and is also typically polynomial.

Furthermore, we introduced the notion of singular multipliers for tangent periodic orbits of billiard. These measure the impact of the singular periodic orbit. In our setting these multipliers play a central role in the dynamics as explained next. We believe billiards with singular periodic orbits are dense among scattering billiards, hence that the concept of singular multipliers may be useful in other investigations of billiard problems. 
While proving the rigorous estimates for the islands size, we have constructed the following local map near singular periodic orbits (12):

$$
\left\{\begin{array}{l}
\bar{u}=v \\
\bar{v}=\xi v+\frac{a \xi}{\sqrt{\kappa}} F(v)-u+(\xi-2) \Gamma .
\end{array}\right.
$$

This map defines universality classes - large classes of different billiard geometries and different potentials give rise to exactly the same local map. The Jacobian of the transformation to this map is $J=\xi a / \delta^{2}$ and the unfolding parameter $\Gamma=v_{\gamma} / \delta$, see Eqs. (25), (26) and (28). Now $\delta$ and $F(\cdot)$ are the rescaling parameter and rescaling function which depend only on the rate at which the smooth billiard potential approaches a billiard - they are independent of the billiard geometry (see Section 2.3 for examples of $\delta, F$ ). Conversely, $\kappa, \xi$, $a$ are parameters which depend only on the billiard flow (the geometry): $\kappa$ is the local curvature near the tangent periodic orbit (local parameter) $\xi$ is the sum of the singular multipliers measuring the instability of the singular periodic orbit (global parameter, independent of the local structure near tangency). The parameter $a$ measures, roughly, how the regular flow rotates the singularity line - the larger the $a$ the closer the image of the singularity line to its normal. The larger the $\xi$ the closer this image is to the unstable eigenvector of the regularized billiard return map, thus for large $\xi$, the quantity $a$ measures the alignment between this eigenvector and the normal to the singularity line.

Summarizing, the coefficient of the nonlinear term in Eq. (17) is given by $(1 / \sqrt{\kappa}) \xi a$ which shows how the curvature, the singular multipliers and the global billiard geometry effects (respectively) scale together to create a significant nonlinear term.

\subsection{Are the islands observable?}

The disappearance of two hyperbolic periodic orbits via a simple singular periodic orbit is a codimension one bifurcation as follows from Section 3.1 of [7]. This implies, in particular, that such a bifurcation persists in one parameter families of scattering billiards. In [7], we conjectured that billiards with simple singular periodic orbits are dense among the scattering billiards; if true, the above observation implies that any one parameter family of scattering billiards must undergo such a bifurcation along a dense set of values of the parameter $\gamma$. Then, by Theorem 1 , islands exist in a $\delta(\epsilon)$ wedge emanating from each such $\gamma$.

Clearly the width of the wedge in parameter space and the size of the island depend on its period $N$. Indeed, examine the map (12). It depends on the two parameters $\xi$ and $\xi a / \sqrt{\kappa}$. For periodic orbits of long period, one expects large singular multipliers hence large $\xi$ : if $\lambda_{\mathrm{L}}$ is the Lyapunov exponent of the billiard map, we expect that for large $N$

$$
\xi=\Lambda+\frac{1}{\Lambda} \approx \exp \left(N \lambda_{\mathrm{L}}\right)
$$

On the other hand, as discussed above, in this limit the parameter $a$ asymptotes a constant positive value. It follows (see Eqs. (32) and (33)) that the stability interval in $\gamma$ values is proportional to $\delta / \xi=\delta(\epsilon) \exp \left(-N \lambda_{\mathrm{L}}\right)$. Finally, recall that the transformation to Eq. (12) involved a Jacobian $\xi\left(1 / \delta^{2}\right) a$, thus, it follows that order one areas in Eq. (12) correspond to order $\delta^{2} \exp \left(-N \lambda_{\mathrm{L}}\right)$ areas in the physical variables $\left(y, y^{\prime}\right)$.

We see that the period of the singular periodic orbit plays a crucial role in the islands' size estimates as well. Consider a value $\gamma^{*}$ for which no singular periodic orbits exist. Consider an $\epsilon$-ball in phase space around a point lying on the singularity line. Using the exponential stretching rates of the billiard, one can argue that in this ball there exists a periodic point of period $N=-2 \ln \epsilon / \lambda_{T}$, where $\lambda_{T}$ denotes the exponential growth rate of lines under the billiard flow. Now, assuming that $\epsilon$ size perturbations of the billiard geometry (i.e., in $\gamma$ ) may be made so that the 
periodic orbit of period $N$ does not hit the corner points (this is another formulation of the density assumption), we obtain that there is a singular periodic orbit of period of order $N$ for a $\gamma$ value which is $\epsilon$-close to $\gamma^{*}$. Summarizing, provided our density conjecture is correct, in an $\epsilon$ ball around $(\gamma, \epsilon)=\left(\gamma^{*}, 0\right)$, for any value of $\gamma^{*}$ there exist a polynomial (in $\epsilon$ ) size set of parameter values for which polynomial size islands of period $\mathrm{O}(\ln \epsilon)$ exist. So, the islands are observable.

\section{Acknowledgements}

We thank H. Primack, Y. Sinai and U. Smilansky for useful discussions. This work has been supported by a research grant from the Henri Gutwirth fund for research. RK also acknowledges the support of the Jakubskind-Cymerman Prize.

\section{Appendix A. The regular map $B_{\epsilon}^{(r)}$}

Here, we prove Lemma 1 regarding the form of the regular auxiliary map. The map $B_{\epsilon}^{(r)}: \Sigma \rightarrow \Sigma$ is constructed by ignoring the passage near the tangent point at the origin, by locally moving the boundary outward as shown in Fig. 4. For the obtained auxiliary billiard the singular periodic orbit $L$ becomes regular. Therefore [7], the map $B_{\epsilon}^{(r)}$ which is the Poincaré map for the auxiliary Hamiltonian flow is $C^{r}$-close to the Poincare map $B_{0}^{(r)}: \Sigma \rightarrow \Sigma$ for the auxiliary billiard flow.

Since $L$ is now regular and the auxiliary billiard is still scattering, the fixed point $\left(y=0, y^{\prime}=0\right)$ of $B_{0}^{(r)}$ at $\gamma=0$ is hyperbolic. Thus, for small $\gamma$ and $\epsilon$ it persists. Denoting the fixed point as $M_{\gamma, \epsilon}=\left(y_{\gamma, \epsilon}, p_{\gamma, \epsilon}\right)$, let us write the Taylor expansion for $B_{\epsilon}^{(r)}$ at $M_{\gamma, \epsilon}$ :

$$
B_{\epsilon}^{(r)}\left(\begin{array}{c}
y \\
y^{\prime}
\end{array}\right)=\left(\begin{array}{l}
y_{\gamma, \epsilon} \\
p_{\gamma, \epsilon}
\end{array}\right)+\left(\begin{array}{ll}
b_{11} & b_{12} \\
b_{21} & b_{22}
\end{array}\right)\left(\begin{array}{c}
y-y_{\gamma, \epsilon} \\
y^{\prime}-p_{\gamma, \epsilon}
\end{array}\right)+\cdots
$$

where the dots stand for nonlinear terms. Now, we establish some inequalities on the coefficients of the linearization matrix $\mathcal{B}=\left\{b_{i j}(\gamma, \epsilon)\right\}$ which will be used in constructing the asymptotic normal form.

The linearization matrix $\mathcal{B}=\left\{b_{i j}(\gamma, \epsilon)\right\}$, depends continuously on the parameters. At $\epsilon=0$, it is the linearization matrix of the Poincaré map for the billiard flow, therefore it must fit the specific hyperbolic structure of the scattering billiard. Thus, it is known [22,23] that the tangent to the unstable manifold of the fixed point $M$ lies in the unstable cone $\mathrm{d} y \cdot \mathrm{d} y^{\prime}>0$ and the tangent to the stable manifold lies in the stable cone $\mathrm{d} y \cdot \mathrm{d} y^{\prime}<0$. Moreover, the unstable cone is mapped inside itself by the linearized map, and the stable cone is mapped inside itself by the inverse to the linearized map. It follows that all the $b_{i j}$ are of same sign, which proves Eq. (19).

In fact, the invariance of the stable and unstable cones of this particular form holds for the map from any crosssection to any other (near each of the cross-sections the $x$-axis must be parallel to the piece of the orbit which is cut by the given cross-section). It is geometrically evident for the scattering billiard that upon each regular reflection with the boundary the orientation is changed on the unstable (as well as on the stable) manifold: after the reflection the positive part of the unstable cone is mapped into the negative one and vice versa. Since the sign of $b_{i j}$ determines whether the linearized map preserves orientation on the unstable manifold of $M$ or not, it follows that

$$
\operatorname{sign} \mathcal{B}=(-1)^{N}
$$

where $N$ is the number of regular collisions of $L$ with the boundary. 


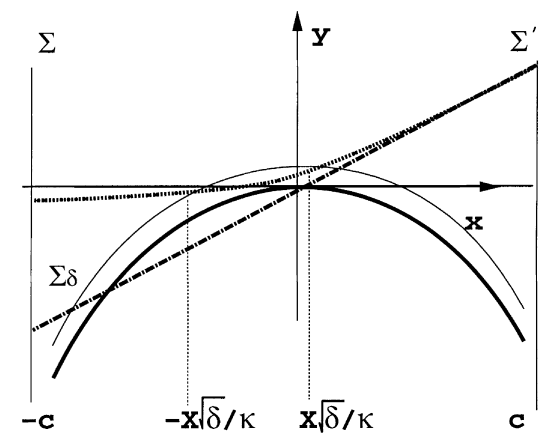

Fig. 7. Details of singular local map construction. ... is the forward singular trajectory of the singular local map, leading to Eq. (B.4). $\cdot-\cdot-\cdot-\cdot-\cdot$ is the auxiliary free backward motion, leading to Eq. (B.5).

At $\gamma=0$ the fixed point $M$ on the cross-section $\Sigma:\{x=-c\}$ is $\left(y=0, y^{\prime}=0\right)$. Infinitesimal increments $\mathrm{d} y$ and $\mathrm{d} y^{\prime}$ which satisfy $\mathrm{d} y+c \mathrm{~d} y^{\prime}=0$, correspond to a family of rays which focus at $(x=0, y=0)$. It is the point of tangency of $L$ to the boundary of the original billiard. For the auxiliary billiard, the boundary is pushed outward near this point. Thus, in the auxiliary billiard, the family of rays pass the focusing point without reflection and becomes divergent. This means that after the first regular reflection the image of the vector $\left(\mathrm{d} y=-c \mathrm{~d} y^{\prime}, \mathrm{d} y^{\prime}\right)$ must belong to the unstable cone. Moreover, it is geometrically evident that it belongs to the same (positive or negative) part of the unstable cone as the image of the vector $\left(\mathrm{d} y=0, \mathrm{~d} y^{\prime}\right)$ for the same $\mathrm{d} y^{\prime}$. This means that (see another proof in [7])

$$
\operatorname{sign}\left(b_{12}-c b_{11}\right)=\operatorname{sign}\left(b_{22}-c b_{21}\right)=\operatorname{sign} \mathcal{B},
$$

namely Eq. (20) holds.

The map $B^{(r)}$ is a measure-preserving diffeomorphism, so

$$
b_{11} b_{22}-b_{12} b_{21}=1 \text {. }
$$

Finally, the trace of the matrix $\left\{b_{i j}\right\}$ is the sum of the multipliers of the hyperbolic fixed point, therefore,

$$
\left|b_{11}+b_{22}\right|>2 \text {. }
$$

\section{Appendix B. The singular map $B_{\epsilon}^{(s)}$}

Here, we prove Lemma 2 regarding the form of the singular map. To construct the singular map, one composes the local forward motion under the full nearly singular flow (see Eq. (B.4)) and the backward motion under the auxiliary Hamiltonian, which amounts to a constant speed motion (see Eq. (B.5)), see Fig. 7. Below, we start with a short set-up of the coordinate system and establish that once Eq. (B.4) is proved we are done. To prove Eq. (B.4), we first integrate the local equations of motion in a boundary layer which is close to the tangency (denoted by $\Sigma_{\delta}$ in Fig. 7). Using rescaling and the rescaling assumption which are valid in $\Sigma_{\delta}$, we obtain simple equations that can be integrated by quadratures (see Eq. (B.13)). We then prove that outside of this boundary layer the motion is essentially free (constant speed motion, see Eqs. (B.15) and (B.16) in the $C^{r}$ topology. This is a non-trivial statement, which is proved by integrating the local equations of motion with the scaled level-set $(\tilde{Q})$ as an independent variable. The composition of the boundary layer motion and the outer free motion produces Eq. (B.4). 
The equations of motion near the boundary are found from Eq. (3):

$$
\begin{array}{ll}
\dot{x}=p_{x}, & \dot{p}_{x}=-W^{\prime}(Q) Q_{x}, \\
\dot{y}=p_{y}, & \dot{p}_{y}=-W^{\prime}(Q) Q_{y} .
\end{array}
$$

Let $\beta(\epsilon, H)$ be defined by the scaling assumption [S]. It is convenient to move the origin onto the level $Q(x, y ; \gamma, \epsilon)=\beta(\epsilon, H)$, to the point where the $x$-axis is tangent to this level (such a point is unique for all small $\epsilon$ and $\gamma$ because the curvature of the boundary never vanishes). Hence:

$$
\left.Q_{x}\right|_{(0,0 ; \gamma, \epsilon)}=0,\left.\quad Q_{y}\right|_{(0,0 ; \gamma, \epsilon)}=1 .
$$

It follows that

$$
Q=\beta+y+\kappa(\gamma, \epsilon) x^{2}+\mathrm{O}\left(x y, y^{2}, x^{3}\right)
$$

where the curvature factor $\kappa$ is strictly positive.

The move of the origin leads to a small shift of the cross-section $\Sigma$ and of the position of the origin on the cross-section; this, obviously, does not affect Proposition 1.

Let us prove that the map from $\Sigma:\{x=-c\}$ to $\Sigma^{\prime}:\{x=c\}$ by the forward flow is written as

$$
y_{\text {out }}=y_{\text {in }}+c\left(y_{\text {in }}^{\prime}+y_{\text {out }}^{\prime}\right)+o(\delta), \quad y_{\text {out }}^{\prime}=y_{\text {in }}^{\prime}+\frac{\delta}{\sqrt{\kappa}} F\left(\frac{y_{\text {in }}+c y_{\text {in }}^{\prime}+o(\delta)}{\delta}\right)+o(\delta),
$$

where 'in' refers to $\Sigma$ and 'out' refers to $\Sigma^{\prime}$; the function $F$ is defined by Eq. (11) and $o(\delta)$ has the same sense as in Lemma 2.

The map $B^{(s)}$ is the composition of Eq. (B.4) and the backward motion from $\Sigma^{\prime}$ to $\Sigma$ with the auxiliary Hamiltonian flow. In the auxiliary Hamiltonian flow the billiard's boundary is pushed outside on a finite distance, so the potential asymptotically vanishes everywhere between $\Sigma^{\prime}$ and $\Sigma$. Therefore, the auxiliary Hamiltonian flow is here $C^{r}$-close to the motion with a constant speed as $\epsilon \rightarrow 0$. Thus, the backward map is written as

$$
\bar{y}_{\text {in }}=y_{0}(\epsilon)+y_{\text {out }}-2 c y_{\text {out }}^{\prime}+o(\delta), \quad \bar{y}_{\text {in }}^{\prime}=p_{0}(\epsilon)+y_{\text {out }}^{\prime}+o(\delta) .
$$

(This is just the Taylor expansion near $\left(y_{\text {out }}, y_{\text {out }}^{\prime}\right)=0$ : since $\left(y_{\text {out }}, y_{\text {out }}^{\prime}\right)=\mathrm{O}(\delta)$ in Lemma 1 , the nonlinearities are $o(\delta)$ in the sense defined there.) The composition of Eqs. (B.4) and (B.5) gives Eq. (22). Thus, to prove the lemma, it is sufficient to prove the formula (B.4).

Notice that $\dot{x} \equiv p_{x}>0$ near the tangent trajectory, thus one may use $x$ as the new independent variable. Fixing the energy implies:

$$
2(H-W(Q, \epsilon))=p_{x}^{2}\left(1+y^{\prime 2}\right)
$$

and Eq. (B.1) are rewritten as

$$
\frac{\mathrm{d} y}{\mathrm{~d} x}=\frac{p_{y}}{p_{x}}, \quad \frac{\mathrm{d}^{2} y}{\mathrm{~d} x^{2}}=-W^{\prime}(Q, \epsilon) \frac{Q_{y}(x, y)-Q_{x}(x, y)(\mathrm{d} y / \mathrm{d} x)}{2(H-W(Q, \epsilon))}\left(1+\left(\frac{\mathrm{d} y}{\mathrm{~d} x}\right)^{2}\right)
$$

Let us rescale the variables, taking into account the smallness of $y$ and $y^{\prime}$ :

$$
y=\delta \tilde{y}, \quad x=\sqrt{\delta} \tilde{x}, \quad \frac{\mathrm{d} y}{\mathrm{~d} x}=\delta p .
$$

From Eq. (B.3) it follows that

$$
Q=\beta+\delta\left(\tilde{y}+\kappa \tilde{x}^{2}+\mathrm{O}(\sqrt{\delta})\right) .
$$


Let us denote

$$
\tilde{Q}=\frac{(Q-\beta)}{\delta}=\tilde{y}+\kappa \tilde{x}^{2}+\mathrm{O}(\sqrt{\delta}) .
$$

Now, the scaling assumption [S] is designed so that Eq. (B.7) becomes, in the rescaled variables,

$$
\begin{aligned}
& \tilde{y}^{\prime}=\sqrt{\delta} p \\
& p^{\prime}=-\frac{1}{2} \tilde{W}_{\epsilon}^{\prime}(\tilde{Q}) G(\tilde{x}, \tilde{y}, p ; \epsilon)
\end{aligned}
$$

where' in the left-hand side denotes the differentiation with respect to $\tilde{x}$; the function $G$ is

$$
G=\frac{Q_{y}(\sqrt{\delta} \tilde{x}, \delta \tilde{y})-\delta Q_{x}(\sqrt{\delta} \tilde{x}, \delta \tilde{y}) p}{1-(\delta /(1-v / H)) \sqrt{\delta} \tilde{W}_{\epsilon}(\tilde{Q})} \frac{1+\delta^{2} p^{2}}{1-v / H} .
$$

For each fixed $(\tilde{x}, \tilde{y}, p)$, as $\epsilon \rightarrow 0$, this system has the following limit (we use Eqs. (B.2) and (B.10)) and that $\nu / H \rightarrow 0$ by the scaling assumption):

$$
\tilde{y}^{\prime}=0, \quad p^{\prime}=-\frac{1}{2} \tilde{W}_{0}^{\prime}\left(\tilde{y}+\kappa \tilde{x}^{2}\right) .
$$

Thus, the solution of Eq. (B.11) approaches the solution of Eq. (B.13) as $\epsilon \rightarrow 0$, on any bounded interval of $\tilde{x}$. It follows that there exists some $X(\epsilon)$ which tends to infinity so slowly that the solution of Eq. (B.11) still approaches (along with all derivatives with respect to the initial conditions) the solution of Eq. (B.13) as $\epsilon \rightarrow 0$, on the unbounded interval $\tilde{x} \in[-C X, C X]$ for any fixed $C$, provided $\tilde{y}$ and $p$ stay bounded (when $\tilde{W}_{0}$ is defined on $R^{+}$only, $\tilde{y}$ should also stay positive and separated from zero).

Take a cross-section $\Sigma_{\delta}=\left\{Q=\beta+\delta X^{2}\right\}=\left\{\tilde{Q}=X^{2}\right\}$, see Fig. 7. By the assumption on $X(\epsilon)$, the system (B.11) inside $\Sigma_{\delta}$ is well approximated by the system (B.13). The latter is easily integrated so one can see that the flow inside $\Sigma_{\delta}$ defines the map $\left(\tilde{y}_{\text {in }, \delta}, p_{\text {in }, \delta}\right) \mapsto\left(\tilde{y}_{\text {out }, \delta}, p_{\text {out }, \delta}\right)$ from any finite size neighborhood of $\tilde{x}=-X / \sqrt{\kappa}$ to a neighborhood of $\tilde{x}=X / \sqrt{\kappa}$, which is written as

$$
\tilde{y}_{\text {out }, \delta}=\tilde{y}_{\text {in }, \delta}+o(1), \quad p_{\text {out }, \delta}=p_{\text {in }, \delta}+\frac{1}{\sqrt{\kappa}} F\left(\tilde{y}_{\text {in }, \delta}\right)+o(1) .
$$

Comparison of Eq. (B.14) with Eq. (B.4) shows that to prove the lemma it is sufficient to verify that the flow outside $\Sigma_{\delta}$ is essentially a constant speed motion. Namely, we will prove that for negative $\tilde{x}$ the flow from $\Sigma$ to $\Sigma_{\delta}$ in the region of finite $\tilde{y}$ and $p$ defines the map

$$
\tilde{y}_{\text {in }, \delta}=\frac{1}{\delta}\left(y_{\text {in }}+c y_{\text {in }}^{\prime}+o(\delta)\right), \quad p_{\text {in }, \delta}=\frac{1}{\delta}\left(y_{\text {in }}^{\prime}+o(\delta)\right),
$$

and for positive $\tilde{x}$ the flow from $\Sigma_{\delta}$ to $\Sigma^{\prime}$ in the region of finite $\tilde{y}$ and $p$ defines the map

$$
y_{\text {out }}=\delta\left(\tilde{y}_{\text {out }, \delta}+c p_{\text {out }, \delta}\right)+o(\delta), \quad y_{\text {out }}^{\prime}=\delta p_{\text {out }, \delta}+o(\delta) .
$$

These two formulas are absolutely symmetric, therefore, we prove only Eq. (B.15). It is convenient to start on the cross-section $\Sigma_{Q}:\{Q(x, y)=Q(-c, 0)\}$ rather than on $\Sigma:\{x=-c\}$. For any orbit which starts with $y_{\text {in }}$ and $y_{\text {in }}^{\prime}$ of order $\mathrm{O}(\delta)$ the distance $\Delta x$ between the intersections with these two cross-sections is $\mathrm{O}(\delta)$ too. The flow near $\Sigma$ is close to the constant speed motion, being on a finite distance on the billiard boundary. Thus, the transition from $\Sigma$ to $\Sigma_{Q}$ adds only $o(\delta)$ terms to $y^{\prime}$, and $y^{\prime} \Delta x$ is added to the value of $y$. Correspondingly, the map $\Sigma \rightarrow \Sigma_{\delta}$ has the form (B.15) if and only if the map $\Sigma_{Q} \rightarrow \Sigma_{\delta}$ is written as follows (we also use the rescaled variables on $\Sigma_{Q}$ ):

$$
\tilde{y}_{\text {in }, \delta}=\tilde{y}_{\text {in }}-\sqrt{\delta} \tilde{x}_{\text {in }} p_{\text {in }}+o(1), \quad p_{\text {in }, \delta}=p_{\text {in }}+o(1)
$$

Let us prove this formula. 
It follows from Eq. (B.3)) that if $c$ is not large, then for all $x \in[-c, c]$

$$
\left|Q_{x}\right| \geq C|x|-\mathrm{O}(y)=C \sqrt{\delta}|\tilde{x}|+\mathrm{O}(\delta)
$$

for some positive constant $C$. Hence

$$
\left|\tilde{Q}^{\prime}\right| \geq \tilde{C}|x|+\mathrm{O}(\sqrt{\delta})
$$

where

$$
\tilde{Q}^{\prime} \equiv \frac{\mathrm{d} \tilde{Q}}{\mathrm{~d} \tilde{x}}=\frac{1}{\sqrt{\delta}} Q_{x}(\sqrt{\delta} \tilde{x}, \delta \tilde{y})+\sqrt{\delta} Q_{y}(\sqrt{\delta} \tilde{x}, \delta \tilde{y}) p .
$$

Recall, that we consider the region of large $\tilde{Q}$ :

$$
X^{2} \equiv Q_{1} \leq \tilde{Q} \leq Q_{2} \equiv \frac{(Q(-c, 0)-\beta)}{\delta},
$$

for finite $\tilde{y}$ and $p$. In this region $\tilde{x}$ is large hence separated from zero so $\tilde{Q}^{\prime} \neq 0$. Thus, $\tilde{Q}$ may be taken as a new independent variable and the equations of motion (B.11) are rewritten as follows:

$$
\frac{\mathrm{d} \tilde{y}}{\mathrm{~d} \tilde{Q}}=\sqrt{\delta} \frac{p}{\tilde{Q}^{\prime}}, \quad \frac{\mathrm{d} p}{\mathrm{~d} \tilde{Q}}=-\frac{1}{2 \tilde{Q}^{\prime}} \tilde{W}_{\epsilon}^{\prime}(\tilde{Q}) G(\tilde{x}, \tilde{y}, p ; \epsilon) .
$$

The variable $\tilde{x}$ is now a function of $\tilde{Q}$ and $\tilde{y}$. For fixed $\tilde{Q}$

$$
\frac{\partial \tilde{x}}{\partial \tilde{y}}=-\frac{\sqrt{\delta}}{Q_{x}} Q_{y}
$$

(see Eqs. (B.8) and (B.10)). It follows from Eq. (B.18) that the ratio $\sqrt{\delta} / Q_{x}(\sqrt{\delta} \tilde{x}, \delta \tilde{y})$ is bounded along with all derivatives with respect to $\tilde{x}$ and $\tilde{y}$. Hence, $\partial \tilde{x} / \partial \tilde{y}$ is bounded, along with all derivatives with respect to $\tilde{y}$, in the region under consideration.

As it is seen from Eq. (B.20), all the derivatives of $\tilde{Q}^{\prime}$ with respect to $\tilde{x}$ and $\tilde{y}$ are bounded. So, it follows now that all the derivatives of $\tilde{Q}^{\prime}$ with respect to $\tilde{y}$ at fixed $\tilde{Q}$ are bounded too. Moreover (see Eqs. (B.19) and (B.10)),

$$
\frac{\partial^{k}}{\partial \tilde{y}^{k}} \frac{1}{\tilde{Q}^{\prime}} \leq \mathrm{const} \cdot \frac{1}{\sqrt{\tilde{Q}}}, \quad k=0, \ldots, r .
$$

When evaluating the solution $(\tilde{y}(\tilde{Q}), p(\tilde{Q}))$ of Eq. (B.22) we are assuming, a prioriy, that it is bounded along with all derivatives with respect to the initial conditions $\left(\tilde{y}_{\text {in }}, p_{\text {in }}\right)$. Namely, we assume that

$$
\|(\tilde{y}(\tilde{Q}), p(\tilde{Q}))\|_{C^{r}} \leq Y
$$

where $Y$ is some sufficiently large constant; we denote as $\|\cdot\|_{C^{r}}$ the maximum of the absolute values of the quantity itself and of all its derivatives with respect to $\left(\tilde{y}_{\text {in }}, p_{\text {in }}\right)$. Note that we do not evaluate the derivatives with respect to $\tilde{Q}$.

This assumption is valid at the starting moment $\left(\tilde{Q}=Q_{2}\right.$, see Eq. (B.21)) so it is, obviously, valid on some interval of values $\tilde{Q}$. Below we show, in particular, that when the estimate (B.25) is satisfied on some interval of $\tilde{Q}$, it is in fact satisfied with the margin of safety; i.e., the actual bound on the $C^{r}$-norm of the solution is independent of the initially assumed value $Y$; see Eq. (B.27). This will justify the a priori assumption for the whole interval $\tilde{Q} \in\left[Q_{1}, Q_{2}\right]$. 
Rewrite the system (B.22)) as

$$
\tilde{y}(\tilde{Q})=\tilde{y}_{\text {in }}+\sqrt{\delta} p_{\text {in }} \int_{Q_{2}}^{\tilde{Q}} \frac{\mathrm{d} q}{\tilde{Q}^{\prime}}+\sqrt{\delta} \int_{Q_{2}}^{\tilde{Q}}\left(p-p_{\text {in }}\right) R \frac{\mathrm{d} q}{\sqrt{q}}, \quad p(\tilde{Q})=p_{\text {in }}-\frac{1}{2} \int_{Q_{2}}^{\tilde{Q}} R G \tilde{W}_{\epsilon}^{\prime}(q) \frac{\mathrm{d} q}{\sqrt{q}},
$$

where $R \equiv \sqrt{\tilde{Q}} / \tilde{Q}^{\prime}$. The functions $R$ and $G$ are uniformly bounded along with all derivatives with respect to $\left(\tilde{y}_{\text {in }}, p_{\text {in }}\right)$. The boundedness follows from Eq. (B.24), from the a prioriy assumed boundedness of $\tilde{y}$ and $p$ (see Eq. (B.25)) and from the boundedness of $Q_{x}, Q_{y}$ and $\partial \tilde{x} / \partial \tilde{y}$. Note also that as $\tilde{Q}$ grows, the function $\tilde{W}_{\epsilon}$ which enters the expression (B.12) for the function $G$ may, in principle, grow unbounded. However, since the integral of $\tilde{W}_{\epsilon^{\prime}} / \sqrt{\tilde{Q}}$ is bounded by assumption $[\mathrm{S}]$, the function $\tilde{W}_{\epsilon}$ is at most linear with respect to $\sqrt{\tilde{Q}}$. Now, since $\tilde{Q}=\mathrm{O}(1 / \delta)$ by definition (see Eq. (B.10)), it follows that the product $\sqrt{\delta} \tilde{W}_{\epsilon}$ is uniformly bounded, so the factor $\left(1-(\delta /(1-v / H)) \sqrt{\delta} \tilde{W}_{\epsilon}(\tilde{Q})\right)^{-1}$ in $G$ is bounded indeed ${ }^{3}$.

Integration of Eq. (B.26) gives (we use that $\sqrt{\delta} \int \frac{\mathrm{d} q}{\sqrt{q}}=\mathrm{O}(\sqrt{\delta} \tilde{Q})$ is bounded since $\tilde{Q}$ is $\mathrm{O}(1 / \delta)$ by definition):

$$
\tilde{y}(\tilde{Q})=\tilde{y}_{\text {in }}+\sqrt{\delta} p_{\text {in }}\left(\tilde{x}-\tilde{x}_{\text {in }}\right)+\mathrm{O}(1) \cdot \max _{q}\left\|p(q)-p_{\text {in }}\right\|_{C^{r}}, \quad p(\tilde{Q})=p_{\text {in }}+\mathrm{O}(1) \cdot \int_{\tilde{Q}}^{Q_{2}}\left|\tilde{W}_{\epsilon}^{\prime}(q)\right| \frac{\mathrm{d} q}{\sqrt{q}}
$$

where $\mathrm{O}(1)$ denotes functions bounded, along with all derivatives with respect to $\left(\tilde{y}_{\text {in }}, p_{\text {in }}\right)$.

By the scaling assumption [S], the integral $\int_{1}^{\infty} \tilde{W}_{\epsilon}^{\prime}(q) \mathrm{d} q / \sqrt{q}$ converges uniformly. Now, since $Q_{1}, Q_{2} \rightarrow \infty$ as $\epsilon \rightarrow 0$, it follows that the integral in the right-hand side of the second equation of (B.27) tends to zero. Thus, though the estimates for the $\mathrm{O}(1)$-factors in Eq. (B.27) may depend on the a priori assumed bound $Y$ for $y$ and $p$, their contribution is canceled by the factor which can be taken arbitrarily small as $\epsilon \rightarrow 0$. This justifies the a priori assumption (B.25).

Putting $\tilde{Q}=Q_{1}$ in Eq. (B.27) gives the map $\Sigma_{Q} \rightarrow \Sigma_{\delta}$. Since $\left|\tilde{x}\left(Q_{1}\right)\right| \leq C X$ and $X(\epsilon)$ is assumed to grow sufficiently slow, the term $\sqrt{\delta} p_{\text {in }} \tilde{x}\left(Q_{1}\right)$ in the first equation of (B.27) is $o(1)$. Thus, we are in a complete agreement with Eq. (B.17). The lemma is proven.

\section{References}

[1] H. Poincaré, Sur les équations de la dynamique et le problème de trois corps, Acta Math. 13 (1890) 1-270.

[2] D.V. Anosov, Geodesic flows on Riemann manifolds with negative curvature, Proc. Steklov Inst. Math., $90,1967$.

[3] Y. Sinai, Dynamical systems with elastic reflections: Ergodic properties of scattering billiards, Russian Math. Sur. 25(1) (1970) 137-189.

[4] Y. Sinai, N. Chernov, Ergodic properties of certain systems of two-dimensional disks and three-dimensional balls, Russ. Math. Surv. 42(3) (1987) 181-207.

[5] G. Gallavotti, D. Ornstein, Billiards and Bernoulli schemes, Comm. Math. Phys. 38 (1974) 83-101.

[6] D. Szász, Boltzmann's ergodic hypothesis, a conjecture for centuries? Studia Sci. Math. Hungar. 31 (1-3) (1996) $299-322$.

[7] D. Turaev, V. Rom-Kedar, Islands appearing in near-ergodic flows, Nonlinearity 11(3) (1998) 575-600.

[8] V. Donnay, Elliptic islands in generalized Sinai billiards, Ergod. Th. Dynam. Sys. 16 (1996) 975-1010.

[9] C. Karney, Long time correlations in the stochastic regime, Physica D 8 (1983) 360.

[10] G. Zaslavsky, M. Edelman, B. Niyazov, Self-similarity, renormalization and phase space nonuniformity of hamiltonian chaotic dynamics, Chaos 7 (1997) 159.

[11] P. Duarte, Plenty of elliptic islands for the standard family of area preserving maps, Ann. Inst. H. Poincaré Anal. Non Liniaire 11(4) (1994) 359-409.

[12] R. MacKay, J. Meiss, I. Percival, Resonances in area preserving maps, Physica D 27 (1987) 1-20.

\footnotetext{
${ }^{3}$ This term depends only on $\tilde{Q}$ and is independent of $\left(\tilde{y}_{\text {in }}, p_{\text {in }}\right)$, so we do not need to estimate its derivatives. This was the main technical reason for introducing $\tilde{Q}$ as a new independent variable.
} 
[13] J. Meiss, E. Ott, Markov tree model of transport in area-preserving maps, Physica D 20 (1986) 387-402.

[14] R. Fleischmann, T. Geisel, R. Ketzmerick, Quenched and negative hall effect in periodic media: application to antidot superlattices, Europhys. lett. 25(3) (1994) 219-224.

[15] G. Zaslavsky, M. Edelman, Maxwell's demon as a dynamical model, Phys. Rev. E 56(5) (1997) 5310-5320.

[16] W.L. Ditto, K. Showalter, Focus issue on control and synchronization of Chaos, Chaos 7(4) (1997) 509-687.

[17] C. Liverani, Decay of correlations, Annals of Mathematics 142 (1995) 239-301.

[18] K. Hansen, Bifurcations and complete chaos for the diamagnetic kepler problem, Phys. Rev. E 51(3) (1995) 1838-1844.

[19] P. Collas, D. Klein, H.-P. Schwebler, Convergence of Hamiltonian systems to billiards, Chaos (1998).

[20] V. Biragov, Bifurcations in a two-parameter family of conservative mappings that are close to the Henon map, Selecta. Mat. Sovietica. 9 (1990) 247-280.

[21] A.I. Neishtadt, V.V. Sidorenko, D.V. Treschev, Stable periodic motions in the problem on passage through a separatrix, Chaos 7(1) (1997) $2-11$.

[22] Y. Sinai, On the foundations of the ergodic hypothesis for dynamical system of statistical mechanics, Dokl. Akad. Nauk. SSSR 153 (1963) $1261-1264$.

[23] M. Wojtkowski, Principles for the design of billiards with nonvanishing lyapunov exponents, Comm. Math. Phys. 105(3) (1986) $391-414$. 